\title{
The Application of Pulp Tissue Derived-Exosomes in Pulp Regeneration: A Novel Cell-Homing Approach
}

\author{
Yan Chen ${ }^{1-5, *}$, Yue $\mathrm{Ma}^{1-5, *}$, Xueting Yang ${ }^{1-5}$, Jinlong Chen ${ }^{1-5}$, Bo Yang $\mathbb{1}^{1-5}$, Weidong Tian ${ }^{1-5}$ \\ 'Engineering Research Center of Oral Translational Medicine, Ministry of Education, West China Hospital of Stomatology, Sichuan University, Chengdu, \\ People's Republic of China; ${ }^{2}$ National Engineering Laboratory for Oral Regenerative Medicine, West China Hospital of Stomatology, Sichuan University, \\ Chengdu, People's Republic of China; ${ }^{3}$ State Key Laboratory of Oral Diseases, West China Hospital of Stomatology, Sichuan University, Chengdu, People's \\ Republic of China; ${ }^{4}$ National Clinical Research Center for Oral Diseases, West China Hospital of Stomatology, Sichuan University, Chengdu, People's \\ Republic of China; ${ }^{5}$ Department of Oral and Maxillofacial Surgery, West China Hospital of Stomatology, Sichuan University, Chengdu, People's Republic of \\ China \\ *These authors contributed equally to this work
}

Correspondence: Weidong Tian; Bo Yang, Department of Oral and Maxillofacial Surgery, West China School of Stomatology, Sichuan University, No. 14, 3rd Section, Ren Min Nan Road, Chengdu, 61004I, People’s Republic of China, Tel/Fax +86 288550 3499, Email dr.yangbo@scu.edu.cn; drtwd@sina.com

Purpose: Exosomes derived from stem cells, as an alternative to stem cells themselves, have been employed for dental pulp regeneration. However, it is not known whether exosomes can recruit host cells to the regeneration process. In this study, we built a "cell homing" model to determine whether exosomes derived from dental pulp tissue (DPT-exos) can regenerate dental pulp by recruiting the stem cells from the apical dental papilla (SCAPs).

Methods: Exosomes were isolated from the dental pulp tissue (DPT-exos) and dental pulp stem cells (DPC-exos) of swine. The effects of the exosomes on SCAPs were compared using CKK-8, Transwell, angiogenesis, and odontogenic induction assays. DPTexos and DPC-exos were investigated in an in vivo "cell homing" model using swine teeth to compare their roles in pulp regeneration. To build the model, we placed SCAP-containing collagen gel at the root tip and filled the cavity of the treated dental matrix (TDM) with DPT-exos and DPC-exos-laden scaffolds, which would be expected to recruit SCAPs to the pulp cavity. The complex was then implanted subcutaneously into immunodeficient nude mice. After eight weeks, tissue samples were taken and analyzed histologically to determine whether the DPT-exos contributed to pulp regeneration through "cell homing".

Results: Exosomes were successfully extracted from dental pulp tissue and confirmed to be exosomes. In vitro tests confirmed that DPT-exos performed better than DPC-exos in promoting the migration, proliferation, and differentiation of SCAPs. Furthermore, DPTexos recruited SCAPs to regenerate dental pulp-like connective tissue in vivo containing collagen, odontoblasts, and enriched predentin-like tissue. Blood vessel growth was demonstrated by immunofluorescence.

Conclusion: This study demonstrated the ability of DPT-exos to induce SCAPs to regenerate connective tissue similar to natural dental pulp. This technique has the potential for treating pulp deficiency caused by various pulp diseases.

Keywords: exosomes, pulp regeneration, cell homing

\section{Introduction}

Although stem cells have been used successfully for the regeneration of dental pulp, ${ }^{1}$ emerging evidence has shown that in vivo transplantation of stem cells carries certain risks, including the induction of myocardial infarction and promoting the development of tumors. ${ }^{2-4}$ Moreover, the clinical application of stem cells also faces restrictions on acquisition, expansion, transportation, and storage. ${ }^{5,6}$ Therefore, the exploration of new therapies to promote dental pulp regeneration using cell-homing techniques has become an urgent need.

Exosomes are a type of extracellular vesicles and can be used in therapeutic applications due to their important roles in intercellular communication. ${ }^{5,6}$ Exosomes have also been used in oral regenerative medicine, specifically, for pulp 
regeneration, and the effectiveness of the technique has been proven. Huang et al found that exosomes secreted by osteoblast-induced dental pulp stem cells (DPSCs) could effectively induce DPSC differentiation in vitro, and could regenerate pulp-like tissue with high expression of odontogenic proteins in nude mice. ${ }^{7}$ Furthermore, in our previous study, we explored the critical role of exosomes isolated from Hertwig's epithelial root sheath cells in pulp regeneration. ${ }^{8}$ In the regenerated pulp tissue, large numbers of odontoblast-like cells, newly formed dental-like tissue, and abundant expression of vascular and neuro-related proteins was observed. These studies demonstrated that exosomes can stimulate the proliferation, migration, odontogenesis, and neurogenesis of odontogenic stem cells as well as angiogenesis, which are the key factors for pulp regeneration, revealing the potential application of exosomes in pulp regeneration and suggesting the usefulness of developing the technique..$^{7-10}$

The above research indicates the positive actions of exosomes on pulp regeneration. However, it is not known whether exosomes can recruit host cells to pulp regeneration through cell homing. In our previous study, we isolated exosomes from adipose tissue (AT-exos) and demonstrated that the AT-exos provided an effective cell-homing approach for producing adipose tissue, offering a successful example of the employment of exosomes derived from tissue in regeneration. ${ }^{11}$ Based on this, our present study employed dental pulp tissue exosomes (DPT-exos) to regenerate dental pulp by cell homing to explore the capacity of tissue regeneration via cell homing. Therefore, in this study, a cell-homing model was developed to test this hypothesis, aiming to find a chairside therapy to regenerate pulp tissue without cell implantation.

To test this hypothesis, we extracted and used DPT-exos while exosomes derived from dental pulp cells (DPC-exos) served as controls. The treated dental matrix (TDM) of swine, $12 \mathrm{~mm}$ in length, was employed to mimic the clinical situation after pulp extraction. For constructing a "cell-homing" model, the TDM was filled with a scaffold loaded with exosomes (DPT-exos or DPC-exos), and stem cells from the apical dental papilla (SCAPs) were placed only at the tip of each root. ${ }^{12}$ This complex was then subcutaneously implanted in nude mice. Eight weeks later, the extent and quality of the newly formed tissue were evaluated. It was found that SCAPs migrated in the canal and formed pulp-like tissue inside the tooth roots. Furthermore, the proliferation and differentiation of the SCAPs, as well as tissue formation, was enhanced by the presence of DPT-exos, including both on the canal walls and scaffold.

\section{Materials and Methods}

\section{Extraction of Teeth from Swine Lower Jaws}

In this study, sufficient pulp tissue is needed to isolate DPT-exos, requiring a large number of teeth. Therefore, deciduous incisor teeth from the mandibles of swines aged 8-12 months were used as the tooth source.These teeth are easily obtained from the slaughterhouse. The pulp tissue was collected from the teeth, cut into small pieces $(\leq 2 \mathrm{~mm})$, sterilized in PBS with $100 \mathrm{U} / \mathrm{mL}$ penicillin and $100 \mu \mathrm{g} / \mathrm{mL}$ streptomycin, and then cultured in serum-free Minimal Essential Medium alpha (MEM- $\alpha$; Hyclone) supplemented with $100 \mathrm{U} / \mathrm{mL}$ penicillin and $100 \mathrm{mg} / \mathrm{mL}$ streptomycin. ${ }^{11,13}$ The culture supernatants were collected after $48 \mathrm{~h}$ for exosome isolation.

\section{Cell Culture}

DPCs were isolated from the pulp of the deciduous incisor of swine as described previously. ${ }^{14}$ To further clarify the boundary between pulp tissues and dental papilla tissues and make them relatively independent, we removed the tissue in the middle part of the two components. Briefly, immediately after extraction, the teeth were cleaned and crushed to access the pulp chamber. After gentle separation from the root, the pulp tissue was thoroughly washed in PBS with antibiotics (as above) and digested with $3 \mathrm{mg} / \mathrm{mL}$ collagenase type I (Hyclone, USA) for $40 \mathrm{~min}$ at $37^{\circ} \mathrm{C}$. The tissue was added to $75 \mathrm{~cm}^{2}$ culture flasks and cultured in MEM- $\alpha$ containing 10\% fetal bovine serum (Hyclone), (L-ascorbic acid2-phosphate, and $1 \%$ penicillin-streptomycin) and cultured under $5 \% \mathrm{CO}_{2}$ at $37^{\circ} \mathrm{C}$. Cells growing from the pulp tissue were cultured under the same conditions. For exosome isolation, DPCs were cultured until $80 \%$ confluent, washed twice with PBS, and cultured in serum-free medium for $48 \mathrm{~h}$. All experiments used passages 3-5 of the cells.

SCAPs were isolated from the apical papilla of the deciduous incisor, as described previously. ${ }^{15}$ Briefly, the root apical papilla was gently separated from the deciduous incisor and was thoroughly washed in PBS containing antibiotics 
(as described above) and digested with $3 \mathrm{mg} / \mathrm{mL}$ collagenase type I (Hyclone, USA) for $40 \mathrm{~min}$ at $37^{\circ} \mathrm{C}$. The tissue was added to $75 \mathrm{~cm}^{2}$ culture flasks and cultured in MEM- $\alpha$ containing $10 \%$ fetal bovine serum (Hyclone), L-ascorbic acid2-phosphate, and $1 \%$ penicillin-streptomycin and cultured under $5 \% \mathrm{CO}_{2}$ at $37{ }^{\circ} \mathrm{C}$. Cells from the apical papilla were cultured under the same conditions.

\section{Isolation and Identification of Exosomes Isolation of Exosomes}

DPT-exos and DPC-exos were purified from the DPT and DPC culture supernatants, by differential centrifugation and ultrafiltration (Supplementary Figure 1). The culture supernatants were centrifuged at $2000 \mathrm{~g}$ for 20 min to remove the cells, followed by centrifugation at $15,000 \mathrm{~g}$ for $40 \mathrm{~min}$ and filtration through a $0.22-\mu \mathrm{m}$ filter to remove cell debris. The material was then passed through an Amicon Ultra-15 Centrifugal Filter Unit with Ultracel-100K (100,000 MW cutoff membrane, Millipore, USA) and centrifuged at $5000 \mathrm{~g}$ for $30 \mathrm{~min}$ to concentrate the two supernatants. The concentrated supernatants were mixed with the Total Exosome Isolation reagent (Life Technologies, USA) at $4^{\circ} \mathrm{C}$ overnight followed by centrifugation at $10,000 \mathrm{~g}$ for $1 \mathrm{~h}$ at $4^{\circ} \mathrm{C}$. The resulting pellets, termed DPT-exos and DPC-exos, respectively, were resuspended in $500 \mu \mathrm{L}$ PBS and the BCA method was used to measure protein concentrations. All steps were conducted at $4{ }^{\circ} \mathrm{C}$. The isolated exosomes were immediately frozen at $-80^{\circ} \mathrm{C}$ until use.

\section{Identification of Exosomes}

A transmission electron microscope (TEM, Hitachi H7500, Japan) was used for examining the exosome ultrastructure. The presence of the exosome markers, CD63 and CD9, was detected by Western blotting. Dynamic light scattering (DLS) was used to measure the size of the exosomes using the Zetasizer Nano ZS90 system (Malvern, UK).

\section{Western Blotting}

The total protein was extracted from vesicles or cells by the Total Protein Extraction Kit (KeyGEN, China), separated on SDS-PAGE, and transferred to polyvinylidene fluoride (PVDF) membranes. The blotted samples were probed with the following antibodies: anti-CD63 (1/1000; Zen-Bio, Durham, NC, USA), anti-CD9 (1/1000; Zen-Bio), and anti-actin (1/ 5000; Abcam, Cambridge, UK). The samples were incubated with the horseradish peroxidase-coupled secondary antibody. Blot images were captured on an ImageQuant LAS 4000 mini system (GE Healthcare Life Sciences, Marlborough, MA, USA) with proteins visualized using the Amersham ECL Select Western blotting detection reagent (GE Healthcare Life Sciences) according to the supplied protocol. Experiments were conducted a minimum of three times.

\section{Biological Effects of DPT-Exos on SCAPs and Human Umbilical Vein Endothelial Cells (HUVECs)}

\section{Uptake of Exosomes by SCAPs and HUVECs}

Exosomes were labeled with Dio (Sigma-Aldrich): $160 \mu \mathrm{g}$ exosomes were diluted in $1 \mathrm{~mL}$ MEM- $\alpha$ with $4 \mu \mathrm{L}$ Dio dye and incubated at $37^{\circ} \mathrm{C}$. After 20 minutes, $500 \mu \mathrm{L}$ Exosome Isolation reagents (Life Technologies, Carlsbad, CA, USA) were added to the solution, incubated at $4^{\circ} \mathrm{C}$ overnight, and centrifuged at $10,000 \mathrm{~g}$ for $1 \mathrm{~h}$ at $4{ }^{\circ} \mathrm{C}$. After removal of the supernatant, the exosome pellet was resuspended in $1 \mathrm{~mL}$ PBS and re-isolated with the reagent for eliminating unbound Dio dye. The labeled exosome pellets were then incubated with SCAPs and HUVECs (iCell Bioscience Inc, Shanghai, China), respectively, for $24 \mathrm{~h}$. After washing, the cells were fixed in 4\% paraformaldehyde for $10 \mathrm{~min}$ and washed again. The nuclei were stained with 6-diamidino-2-phenylindole (DAPI). Images were captured by confocal microscopy (Olympus FV1200, Olympus).

\section{Cell Proliferation Assays}

The proliferation of SCAPs was measured with the Cell Counting Kit-8 (Dojindo, Japan). SCAPs were seeded $\left(1 \times 10^{3}\right.$ cells/well) into 96-well plates and incubated overnight at $37^{\circ} \mathrm{C}$. The cells were then cultured in MEM- $\alpha$ containing DPCexos and DPT-exos at 10,40, and $160 \mu \mathrm{g} / \mathrm{mL}$ respectively, while the blank control contained medium only without exosomes. At 1, 3, 5, 7, 9, and 11 days, $10 \mu \mathrm{L}$ of the CCK-8 solution was added to each well and allowed to incubate at $37^{\circ} \mathrm{C}$ for 90 mins. OD values were measured with a Multiskan Go Spectrophotometer (Thermo Fisher Scientific, 
Waltham, MA, USA). HUVEC proliferation was determined in the same way using the time points of 1, 2, 3, and 4 days. All experiments were conducted at least three times.

\section{Cell Migration Assays}

SCAP migration was measured using Transwell assays. Briefly, $1 \times 10^{4}$ cells were loaded into the upper compartment of a Chemotaxicell Chamber ( $8 \mu \mathrm{m}$, Osaka, Japan) and 500 $\mu \mathrm{L}$ MEM- $\alpha$ containing 5\% FBS with DPC-exos and DPT-exos $(160 \mu \mathrm{g} / \mathrm{mL})$, respectively, were added to the lower compartment while medium without exosomes was used as the control. After 24 hours, the chamber was washed with PBS. Cells that had not migrated were detached from the membrane's upper side using a cotton swab and the remaining cells were fixed as above and stained with $0.5 \%$ crystal violet for $10 \mathrm{~min}$. Cells were counted in the middle, top, bottom, left, and right microscopic fields per filter and divided into 5 to obtain the number of migrated cells. HUVEC migration was determined using the same method. All experiments were carried out at least three times.

\section{Tube Formation Assay as a Measure of Angiogenesis}

HUVECs were pretreated with $160 \mu \mathrm{g} / \mathrm{mL}$ DPT-exos and DPC-exos, respectively, for 2 days, then collected, and seeded at $10^{4}$ cells per well into 96-well plates coated with Matrigel. ${ }^{8}$ Phase-contrast images were acquired after $6 \mathrm{~h}$ using an inverted microscope (Olympus, Japan). The numbers of nodes and the lengths of tubes in each field were determined with Image Pro Plus software. All experiments were conducted at least three times.

\section{Alkaline Phosphatase Assay}

To measure alkaline phosphatase (ALP), DPCs were cultured with or without exosomes. At day 4, ALP activity was measured using the ALP KIT (Beyotime, China). In addition, a second ALP kit (Jiancheng, China) was used and the activity was normalized to the protein concentration. Absorbances at $520 \mathrm{~nm}$ were measured using the Multiskan Go Spectrophotometer. All experiments were conducted at least three times.

\section{Mineralization Assays}

SCAPs were inoculated at $5 \times 10^{4}$ cells per well into 24 -well plates and divided into three groups. DPC-exos $(160 \mu \mathrm{g} / \mathrm{mL})$ and DPT-exos $(160 \mu \mathrm{g} / \mathrm{mL})$ were used to compare their ability to promote mineralization in osteogenic medium (OM, consisting of basal medium, $0.01 \mu \mathrm{M}$ dexamethasone, $50 \mu \mathrm{g} / \mathrm{mL}$ ascorbic acid, $0.01 \mu \mathrm{M}$ dihydroxyvitamin-D3, and 10 $\mathrm{mM}$ glycerophosphate). Mineralization was determined by Alizarin Red staining after 5 days' incubation, while calcium concentrations were determined by $562 \mathrm{~nm}$ absorbance after destaining the mineralized bone nodules with $10 \%$ cetylpyridinium chloride. At least three technical replicates were obtained.

\section{Cells and Exosomes Loaded on Collagen Gels}

For determining whether collagen gel could be employed to carry exosomes and SCAPs in vivo, the DiO-labeled exosomes $(2 \mathrm{mg} / \mathrm{mL})$ were combined with collagen gels, and the presence of exosomes on the gels examined under a confocal microscope (Olympus FV1200, Olympus, Japan). SCAPs were mixed with DPT-exos and DPC-exos, respectively $(160 \mu \mathrm{g} / \mathrm{mL})$, and collagen gel, while the control contained only cells with the collagen gel. After incubation for 1 and 7 days, the viability and adhesion of the cells were examined with confocal microscopy after staining cells with the live/dead assay kit (keyGEN BioTECH). This uses calcein AM (green) and propidium iodide (red) to stain live and dead cells, respectively. All experiments were conducted at least three times.

\section{Preparation of Treated Dentin Matrix}

TDM was prepared as previously described. ${ }^{16}$ Briefly, incisors were extracted from the jaws of swine, with the upper parts fashioned using a sterile fissure bur to a $12 \mathrm{~mm}$ height and $2 \mathrm{~mm}$ internal diameter following the removal of the enamel, bone, and cementum on the surface of the teeth. The dentin tubes were soaked in $17 \%$ ethylenediamine tetraacetic acid (EDTA) for $10 \mathrm{~min}$ at room temperature to eliminate the smear layer, and treated with iodophor and $5.25 \%$ $\mathrm{NaClO}$ for $30 \mathrm{~min}$ and $10-15 \mathrm{~min}$, respectively. After washing in sterile $\mathrm{PBS}$, the tubes were incubated at $37^{\circ} \mathrm{C}$ in antibiotic-containing PBS for 3-7 days to eliminate disinfectant residue and possible bacterial contamination. 


\section{Animals}

All animal experiments were performed according to protocols approved by the Institutional Animal Care and Use Committee (IACUC) at Sichuan University. This study was reviewed and approved by the Committee of Ethics of West China Hospital of Stomatology, Sichuan University (NO. WCHSIRB-D-2021-033).

Twenty female nude mice (6 weeks old) were obtained from Jiangsu GemPharmatech Co. Ltd.

\section{In vivo Implantation in Immunodeficient Nude Mice}

Exosomes and SCAPs were mixed with collagen gel and then used to fill the pulp canal of the dentine tube in a stratified manner for constructing the cell-homing model. According to the composition of the filling, all the samples were divided into five groups $(\mathrm{n}=3)$ (Supplementary Figure 2): (1) the DPT-exos+SCAPs+Col-I, test group, in which the TDM was filled with collagen gel with DPT-exos and with SCAPs at the root tips; (2) the DPC-exos+SCAPs+Col-I, control group, in which the TDM was filled with collagen gel with DPC-exos and with SCAPs at the root tips; (3) the DPT-exos+Col-I, control group, in which the TDM was filled with collagen gel and with DPC-exos; (4) the SCAPs+Col-I, control group, in which the TDM was filled with collagen gel without exosomes and with SCAPs at the root tips; (5) Col-I, blank group, in which the TDM was filled only with collagen gel. The samples were subcutaneously injected into the left and right sides of the back. Mice were aged between 6 and 8 weeks old. After eight weeks, the grafts were removed, fixed with $4 \%$ paraformaldehyde, decalcified with $10 \%$ buffered EDTA, and paraffin-embedded. Sections of $6 \mu \mathrm{m}$ were made for subsequent histological and immunological examinations. All experiments were carried out at least three times.

\section{Hematoxylin/Eosin Staining}

The fixed tissue was dehydrated using an ethanol gradient $(50 \%, 70 \%, 95 \%$, and $100 \%$ ethanol), cleared with xylene, and paraffin-embedded. Six-micrometer sections were cut, stained with hematoxylin/eosin (H\&E), and examined under light microscopy. Images were combined to show the entire pulp cavity.

\section{Immunofluorescent Staining}

Immunofluorescent (IF) staining was carried out as previously described ${ }^{17}$ The primary antibodies were anti-MBP101 (1:200; Abcam), anti-NF200 (1:200; Abcam), anti-VEGF (1:200; Abcam), and anti-CD31 (1:200; Abcam). Goat antirabbit 488 (1/200; Invitrogen) and goat anti-mouse 488 (1/200; Invitrogen) secondary antibodies were used. Sections and cells were mounted in fluorescent mounting medium after DAPI counterstaining, and were examined under a fluorescence confocal microscope (Olympus FV1200).

\section{Immunohistochemical (IHC) Staining}

Primary antibodies against DSPP (1:200; Zen-Bio), DMP1 (1:200; Biovision), ALP (1:200; Abcam), and DSP (1:200; Santa Cruz) were used. PBS alone was used as a negative control. Visualization of secondary antibody staining was performed using the DAB kit (Gene Tech, China). The experiment was repeated three times.

\section{Statistics}

All data were expressed as mean \pm standard deviation (SD). Significant differences were assessed by one-way ANOVA for three groups. $\mathrm{P}<0.05 * \mathrm{P}<0.01 * * \mathrm{P}<0.001 * * *$ and $\mathrm{P}<0.0001 * * * *$ were considered to be statistically significant and ns represented non-significance.

\section{Results and Discussion Characterization of Exosomes}

To test whether the isolated vesicles were exosomes, we used TEM, Western blotting, and dynamic light scattering measurements to observe the shape, surface markers, and diameters of the structures. As shown in Figure 1, both DPTexos and DPC-exos presented typical cup-like shapes with diameters smaller than $150 \mathrm{~nm}$ determined by TEM and DLS (Figure 1A and B). Both DPT-exos and DPC-exos expressed the exosomal markers CD63 and CD9, while the cellular marker actin was not detected (Figure 1C). These results indicate that the DPT-exos conformed to the biological characteristics of 
A

DPT-exos

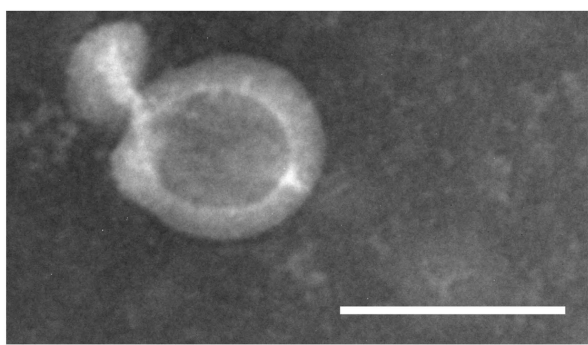

B

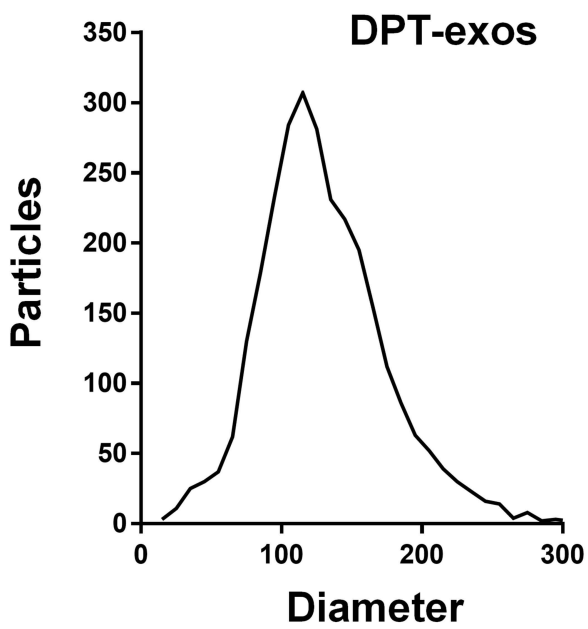

C



Actin

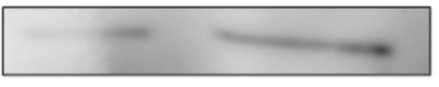

물

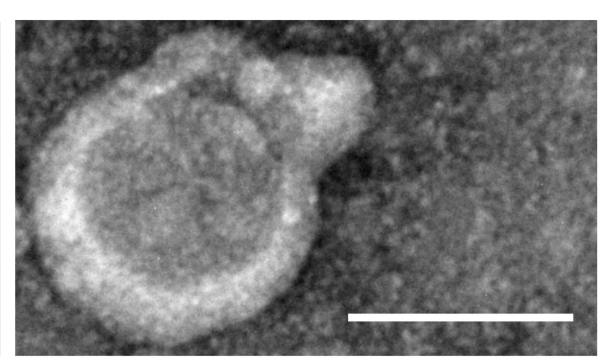

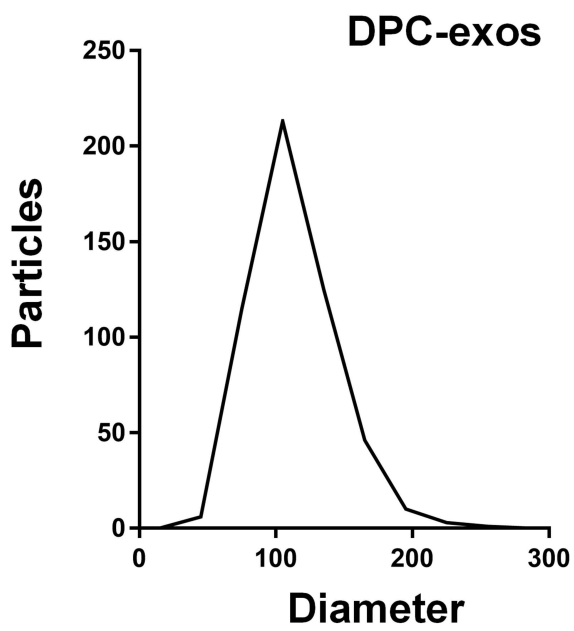

DPC-exos
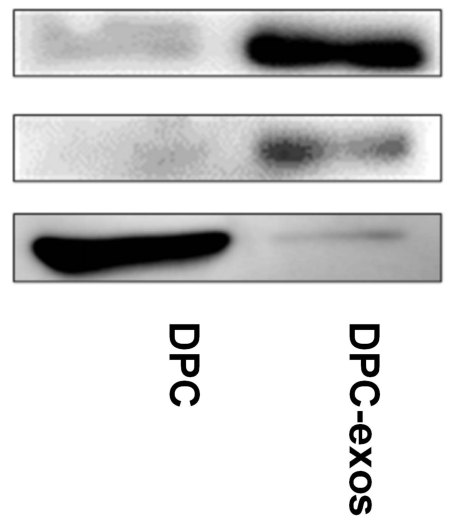

Figure I Identification of exosomes. (A) The morphology of DPT-exos and DPC-exos; (B) the diameter distribution of DPT-exos and DPC-exos; (C) surface markers of DPT-exos and DPC-exos. Scale bars: $100 \mathrm{~nm}$.

exosomes and could be utilized to further research. In addition, some CD9 was observed in the DPT-exos, which does not agree with other studies, indicating that the extraction method we used has some limitations. However, there are no reports on extraction methods that can guarantee the purity of tissue-derived exosomes, ${ }^{12}$ an issue that needs further exploration.

\section{Biological Effects of DPT-Exos on SCAPs and HUVECs} Endocytosis of Exosomes by SCAPs and HUVECs

To investigate whether DPT-exos and DPC-exos were internalized by SCAPs and HUVECs, cells were incubated with DiO-labeled DPT-exos and DPC-exos for 6 hours. Immunofluorescent staining showed that DiO-labeled DPT-exos and 
DPC-exos surrounded the nuclei in the cells after internalization (Figure 2A), indicating successful endocytosis. The result laid a foundation for further research on the effects of exosomes on SCAPs and HUVECs.

\section{Exosomes Promote Proliferation and Migration of SCAPs and HUVECs}

The CCK-8 assay showed that, although the OD values of both the DPT-exos and DPC-exos groups increased with increasing exosome concentration compared with the blank group, the ODs of the DPT-exos groups were significantly higher than the DPC-exos groups (Figure 2B). A similar result was also observed in the HUVEC proliferation assay, while the differences between the groups were hard to detect on day 4 (Figure $2 \mathrm{~B}$ ). This suggested that the DPT-exos promoted proliferation more strongly than the DPC-exos in both cell types, with $160 \mu \mathrm{g} / \mathrm{mL}$ found to be the optimal concentration for both DPT-exos and DPC-exos. This may be related to the AKT and ERK pathways promoted by exosomes. ${ }^{18}$ This exosome concentration was, therefore, used for the following assays.

The migration experiment showed that when exosomes were included in the lower chamber, migration of both types of cells increased in comparison to the blank group (Figure 2C and D). Both DPT-exos and DPC-exos induced cell migration, although the rate of migration did not differ significantly between the two types of exosomes. As the success of cell-homing tissue regeneration depends on whether the host cells can be recruited to the defect area, both cell proliferation and migration play important roles. ${ }^{5}$ These results demonstrate that DPT-exos significantly induce both the proliferation and migration of SCAPs and would be expected to recruit SCAPs to migrate to the pulp cavity. Ivica et al demonstrated that exosomes can induce the recruitment and proliferation of human mesenchymal stem cells, while Xian et al found that exosomes play important roles in angiogenesis through the promotion of proangiogenic factor expression and endothelial cell proliferation. These results are consistent with the ability of DPT-exos to promote cell migration, proliferation, and angiogenesis observed in this study. ${ }^{10,19}$

\section{Exosomes Induce Odontogenic Differentiation of SCAPs}

Exosomes are expected to modify the function of host cells. ${ }^{20}$ In this case, we treated SCAPs with DPT-exos and investigated osteogenic induction. After 5 days of co-culture, increased levels of ALP and the formation of mineralized nodules were observed (Figure 3A and B). This suggested that DPT-exos had a positive effect on promoting biomineralization, which is
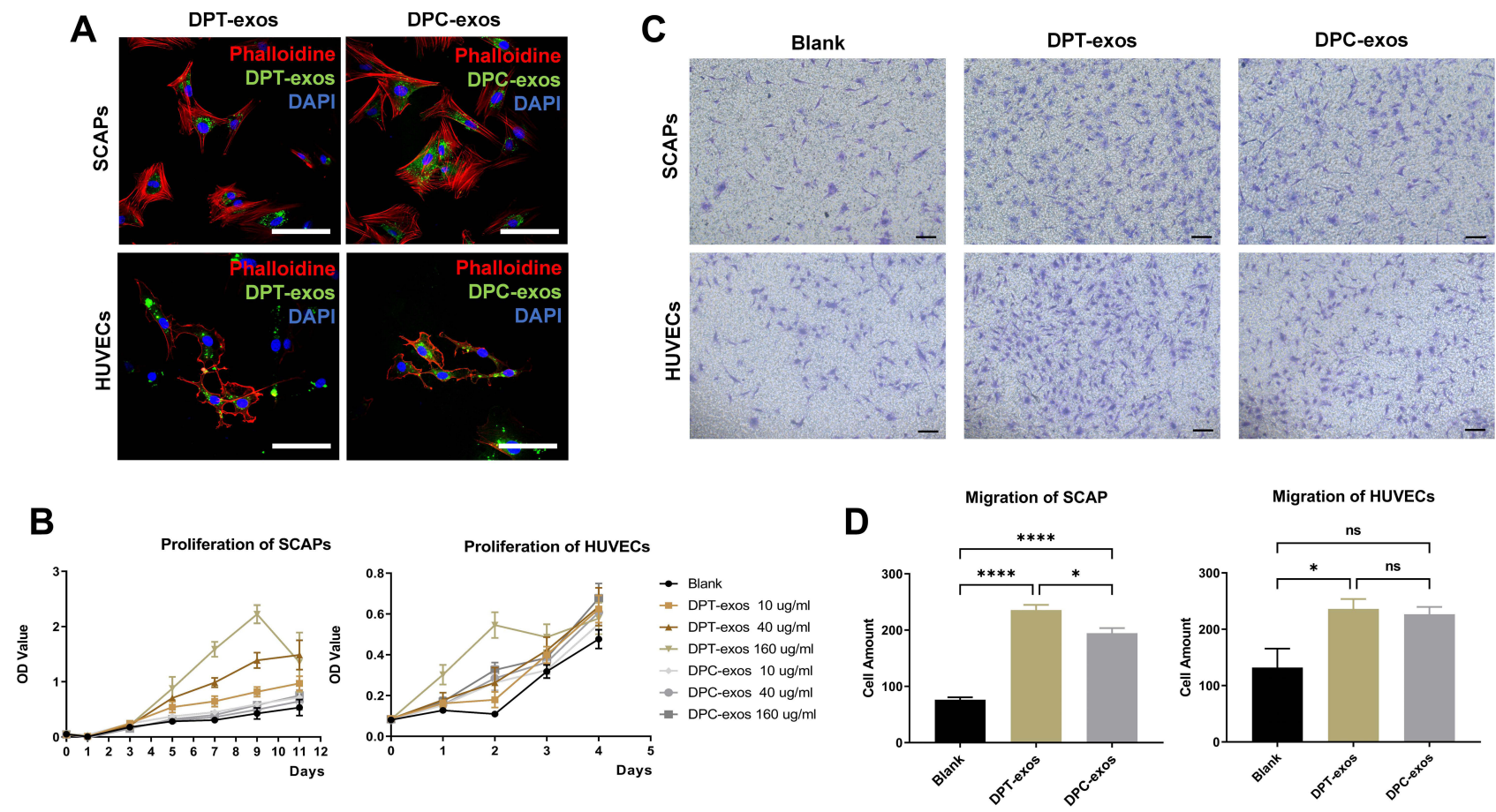

Figure 2 Exosomes promote proliferation and migration of SCAPs and HUVECs. (A) The uptake of DPT-exos and DPC-exos by SCAPs and HUVECs, respectively. (B) The proliferation of SCAPs and HUVECs measured by the CCK-8 assay using the optimal concentration of $160 \mu g / \mathrm{mL}$ DPT-exos and DPC-exos. (C) Cell migration shown by the Transwell assay. (D) Migrated cells in different fields of view from the Transwell assay. *P=2.35L $f^{-2}$, ****P<IL $f^{4}$, using one-way ANOVA. Scale bars: (A) $50 \mu \mathrm{m}$, (C) I00 $\mu \mathrm{m}$. 
A

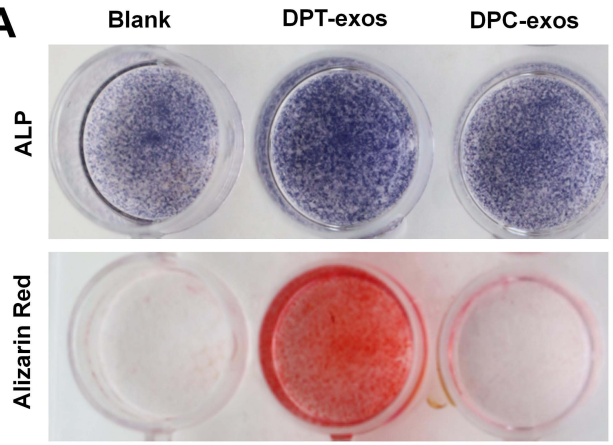

C

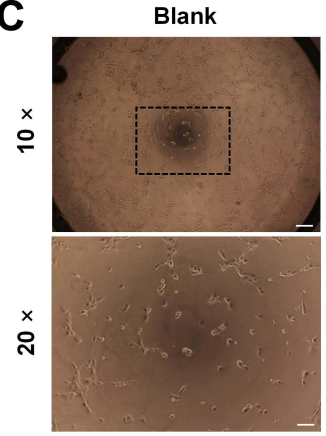

DPT-exos

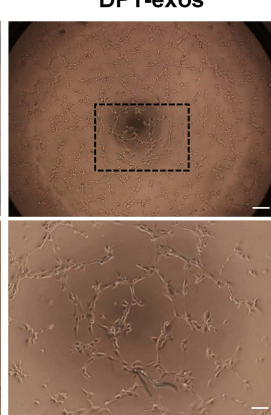

DPC-exos

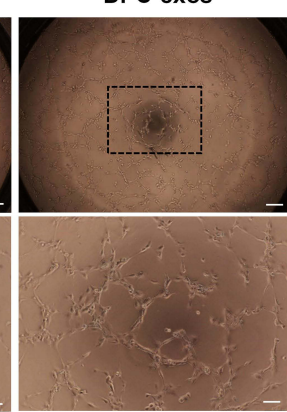

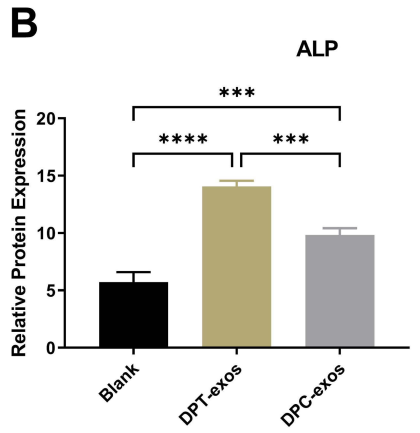
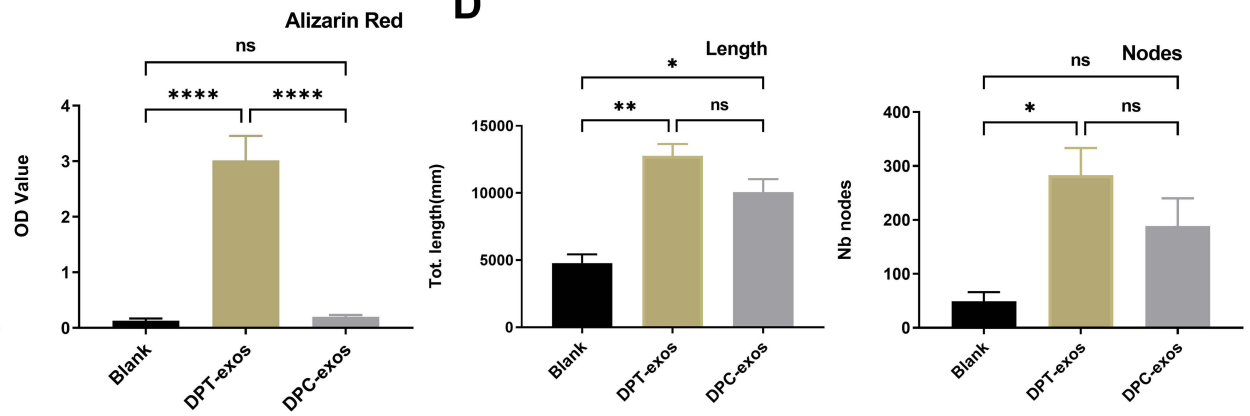

Figure 3 Exosomes promote SCAP differentiation and tube formation. (A) Representative images of ALP and Alizarin Red S staining. (B) Quantitation of ALP activity

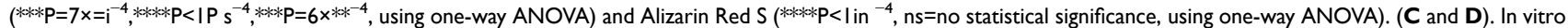
tube formation of HUVECs and lengths of tubes $\left(* \mathrm{P}=1.09 \times 10^{-2}, * * \mathrm{P}=1.4 \times 10^{-3}\right.$, ns $=$ no statistical significance, using one-way ANOVA) and total number of nodes $\left(* \mathrm{P}=1.9 \times 10^{-2}\right.$, ns=no statistical significance using one-way ANOVA) and up-regulation after treatment with DPT-exos (I60 $\left.\mu g / \mathrm{mL}\right)$ and $\mathrm{DPC}$-exos $(160 \mu \mathrm{g} / \mathrm{mL})$. Scale bars: (A) $1 \mathrm{~cm}$, (C) $200 \mu \mathrm{m}$ and $100 \mu \mathrm{m}$.

a critical process in osteogenesis and dentin formation. Therefore, DPT-exos would be expected to accomplish dentin regeneration through SCAP induction, which is an indispensable part of the normal physiological function of dental pulp. ${ }^{21,22}$

ALP and mineralized nodule tests, however, do not directly account for odontogenesis. The mechanism of dentine formation is related to mineralization. According to previous experiments, ALP and mineralized nodules can explain the possibility of odontogenic differentiation to a certain extent. ${ }^{23-25}$ Therefore, we considered whether DPT-exos had the ability to promote mineralization through in vitro experiments, thus determining the feasibility of subsequent experiments investigating the odontogenic ability of DPT-exos in vivo.

\section{Exosomes Promote Angiogenesis of HUVECs}

Vascularization is a precondition for pulp regeneration as it provides nutrition. Therefore, we evaluated the potential action of DPT-exos and DPC-exos on angiogenesis. The cells were seeded on Matrigel and were then co-cultured with DPT-exos or DPC-exos, using PBS as a negative control. After four hours' incubation, we observed markedly increased tube formation in the exosome-treated cells in comparison with the control (Figure 3C). The total tube lengths and number of nodes were measured (Figure 3D), showing that DPT-exos and DPC-exos notably enhanced the cells' ability to form tubes and that DPT-exos performed better than DPC-exos. These results provide a foundation for follow-up studies on whether DPT-exos can promote pulp vascularization in vivo, which is the key to pulp regeneration.

\section{Distribution of SCAPs and Exosomes in Collagen Gels}

Collagen gel was employed to load exosomes and SCAPs separately and its loading capacity was further investigated by confocal microscopy. After resuspension with the collagen gel, the DiO-labeled exosomes were observed to be distributed uniformly on the gel, demonstrating binding of the exosomes to the gel (Supplementary Figure 3A). According to the Live/Dead Assay, the SCAPs in the gel showed a high degree of viability and the results revealed that DPT-exos can increase the proliferation of SCAPs after 1 to 7 days' culture (Supplementary Figure 3B). 


\section{DPT-Exos Recruit SCAPs from the Pulp Apex to Regenerate Pulp-Like Tissue}

After ensuring that the recruitment and differentiation of SCAPs can be triggered by DPT-exos in vitro, we conducted ectopic experiments to further explore its potential for cell homing in pulp regeneration. To do this, we constructed an in vivo implant model based on the TDM. The SCAPs-laden gel and DPT-exos-laden gel filled in layers into the TDM cavity, as presented in Supplementary Figure 2A, to simulate the distribution of stem cells in the dental pulp after tooth eruption. We used five groups to explore the potential of DPT-exos to regenerate pulp by recruiting SCAPs (Supplementary Figure 2B): (1) DPT-exos+SCAPs+Col-I, test group, in which pig TDM was filled with collagen gel and DPT-exos, and with SCAPs at the root tips; (2) DPC-exos+SCAPs+Col-I, control group, in which the TDM was filled with collagen gel and DPC-exos, and with SCAPs at the root tips; (3) DPT-exos+Col-I, control group, in which the TDM was filled with collagen gel with only DPC-exos; (4) SCAPs+Col-I, control group, in which the TDM was filled with collagen gel without exosomes and with SCAPs at the root tips; (5) Col-I, blank group, in which the TDM was only filled with collagen gel.

Two months after implantation, new tissue formation was visible in the cavities of every group. However, compared with the other groups, the regenerated pulp-like tissues in the DPT-exos+SCAPs+Col-I group filled almost the entire cavity. In addition, we observed predentin-like tissue, numerous blood vessels, and polarizing odontoblast-like cells as well as dense collagen fibers in the new tissue (Figure 4). Furthermore, dentin-like mineralization and polarizing odontoblast-like cells were observed in the DPT-exos+SCAPs+Col-I group, whereas only a small amount of mineralized tissue was seen in the DPC-exos+SCAPs+Col-I group. The remaining three groups showed only extracellular matrix and collagen deposition concentrated at the apex of the root (Supplementary Figure 4).

To further define whether the SCAPs in the DPT-exos+SCAPs+Col-I group had differentiated into odontoblast-like cells and produced dentin-like mineralization, IHC was used to examine the expression of odontogenic proteins (ALP, DSP, DMP-1, and DSPP). As seen in Figure 5, both DMP-1 and DSPP were strongly expressed on the dentin-like mineralization connected to the mature dentine, while ALP and DSP expression was seen on polarizing odontoblast-like cells attached to the dentin-like mineralization (Figure 5A). As expected, the DPT-exos had a positive effect on promoting odontogenic differentiation, consistent with the in vitro experiments. These are novel findings and suggest
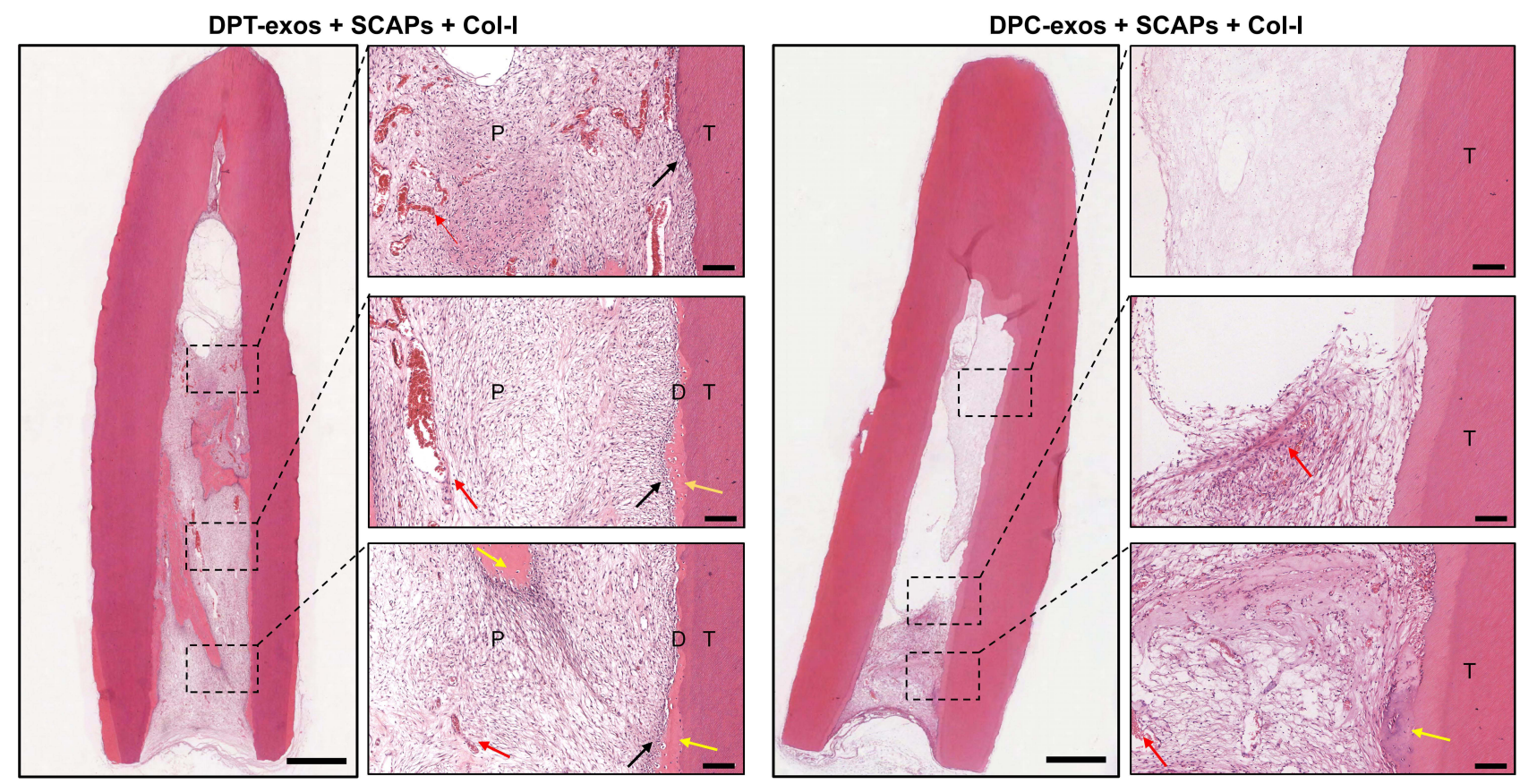

Figure 4 DPT-exos induce the regeneration of pulp-like tissue. H\&E staining showing odontoblast-like cells (black arrows), rich blood vessels (red arrows), and regenerated dentin-like tissue (blue arrows) at the interface between the dentin and pulp-like tissue in the DPT-exos+SCAPs+Col-I group. Only sparse blood vessels (red arrows) and immature regenerated dentin-like tissue were seen in DPC-exos+SCAPs+Col-I group. Scale bars: I mm and $100 \mu \mathrm{m}$.

Abbreviations: T, treated dentin matrix; D, dental-like tissue; P, pulp-like tissue. 

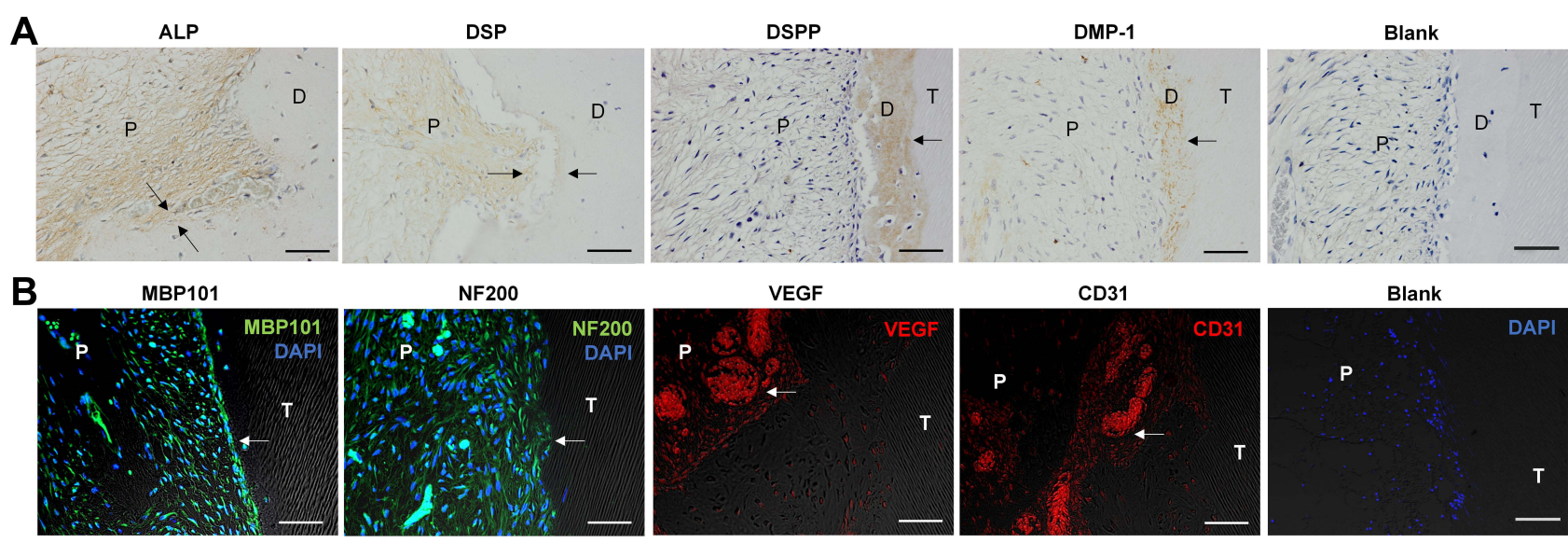

Figure 5 DPT-exos enhance odontogenic and neurogenetic differentiation of SCAPs and revascularization. (A) IHC staining showing positive expression of odontoblastic markers (ALP, DSP, DSPP, DMPI, Blank) in the DPT-exos+SCAPs+Col-I (black arrows). (B) Immunofluorescence showing positive expression of angiogenic markers (CD3I and VEGF) and neurogenetic markers (MBPI0I and NF200) in the DPT-exos+SCAPs+Col-I group (white arrows). The PBS negative control did not produce staining in the harvested tissues (Blank). Scale bars: $50 \mu \mathrm{m}$.

Abbreviations: ALP, alkaline phosphatase; DSP, dentin sialoprotein; DSPP, dentin sialophosphoprotein; DMPI, dentin matrix protein I; Blank, the PBS negative control; T, treated dentin matrix; $\mathrm{D}$, dental-like tissue; $\mathrm{P}$, pulp-like tissue.

the potential of exosome application in pulp regeneration. ${ }^{7,8}$ However, only fiber adsorption staining of the extracellular matrix was found in the other groups (Supplementary Figures 5 and $\underline{6}$ ).

Neurovascularization is a key process in pulp regeneration and also forms the basis of natural pulp function. ${ }^{26,27}$ The vascular system provides nutrients and removes waste from the pulp, as well as contributing significantly to inflammation and regeneration of the pulp. IF staining showed that the newly formed tissues in the PT-exos+SCAPs + Col-I group strongly expressed the angiogenic markers CD31 and VEGF (Figure 5B), clearly distributed around the blood vessels, which is consistent with the H\&E staining. Nerves also perform sensory functions in the pulp and are responsible for responding to external stimuli. Raised levels of the neurogenetic markers MBP101 and NF200 were seen in the DPT-exos+SCAPs+Col-I group in the immunofluorescence experiments, similar to the native group (Figure 5B, Supplementary Figure 6). The colored cells appear to be dendritic cells, similar to nerve cells. In DPCexos+SCAPs+Col-I group and DPT-exos+Col-I group, only a small number of tiny nerve filaments and small blood vessels were observed. In contrast, no significant expression of specific proteins related to neurogenesis and angiogenesis was observed in SCAPs+Col-I group and Col-I group (Supplementary Figure 6). These results indicate the great potential of DPT-exos in pulpal vascularization and neuralization, further confirming its ability in pulp regeneration.

\section{Conclusion}

In conclusion, this study used DPT-exos to induce SCAPs to regenerate connective tissue similar to natural pulp, demonstrating the ability of DPT-exos to recruit SCAPs into the pulp cavity for pulp regeneration. In addition, this study demonstrated the ability of DPT-exos to promote blood vessel and nerve formation during pulp regeneration, which is crucial in pulp regeneration therapy. Thus, DPT-exos have the potential to be applied to the treatment of pulp deficiency caused by various pulp diseases.

\section{Ethics Approval and Informed Consent}

All animal experiments were performed according to protocols approved by the Institutional Animal Care and Use Committee (IACUC) at Sichuan University. This study was reviewed and approved by the Committee of Ethics of West China Hospital of Stomatology, Sichuan University (NO. WCHSIRB-D-2021-033). 


\section{Acknowledgments}

This work is supported by National Key Technology R\&D Program (2017YFA0104800) and the Sichuan Science and Technology Program (2017SZ0031).

\section{Disclosure}

The authors report no conflicts of interest related to this study.

\section{References}

1. Xuan K, Li B, Guo H, et al. Deciduous autologous tooth stem cells regenerate dental pulp after implantation into injured teeth. Sci Transl Med. 2018;10:3227. doi:10.1126/scitranslmed.aaf3227

2. Vulliet PR, Greeley M, Halloran SM, et al. Intra-coronary arterial injection of mesenchymal stromal cells and microinfarction in dogs. Lancet. 2004;363:783-784. doi:10.1016/S0140-6736(04)15695-X

3. Shi Y, Du L, Lin L, et al. Tumour-associated mesenchymal stem/stromal cells: emerging therapeutic targets. Nat Rev Drug Discov. $2017 ; 16: 35-52$. doi:10.1038/nrd.2016.193

4. Yan X, Zhang D, Wu W, et al. Mesenchymal stem cells promote hepatocarcinogenesis via lncRNA-MUF interaction with ANXA2 and miR-34a. Cancer Res. 2017;77:6704-6716. doi:10.1158/0008-5472.CAN-17-1915

5. Eramo S, Natali A, Pinna R, et al. Dental pulp regeneration via cell homing. Int Endod J. 2018;51:405-419. doi:10.1111/iej.12868

6. Ivica A, Zehnder M, Weber FE. Therapeutic potential of mesenchymal stem cell-derived extracellular vesicles in regenerative endodontics. Eur Cell Mater. 2021;41:233-244. doi:10.22203/eCM.v041a17

7. Huang CC, Narayanan R, Alapati S, et al. Exosomes as biomimetic tools for stem cell differentiation: applications in dental pulp tissue regeneration. Biomaterials. 2016;111:103-115. doi:10.1016/j.biomaterials.2016.09.029

8. Zhang S, Yang Y, Jia S, et al. Exosome-like vesicles derived from Hertwig's epithelial root sheath cells promote the regeneration of dentin-pulp tissue. Theranostics. 2020;10:5914-5931. doi:10.7150/thno.43156

9. Hu X, Zhong Y, Kong Y, et al. Lineage-specific exosomes promote the odontogenic differentiation of human dental pulp stem cells (DPSCs) through TGFß1/smads signaling pathway via transfer of microRNAs. Stem Cell Res Ther. 2019;10:170. doi:10.1186/s13287-019-1278-x

10. Xian X, Gong Q, Li C, et al. Exosomes with highly angiogenic potential for possible use in pulp regeneration. $J$ Endod. 2018;44:751-758.

11. Dai M, Yu M, Zhang Y, et al. Exosome-like vesicles derived from adipose tissue provide biochemical cues for adipose tissue regeneration. Tissue Eng Part A. 2017;23:1221-1230. doi:10.1089/ten.tea.2017.0045

12. Widbiller M, Driesen RB, Eidt A, et al. Cell homing for pulp tissue engineering with endogenous dentin matrix proteins. $J$ Endod. 2018;44:956-962. doi:10.1016/j.joen.2018.02.011

13. Théry C, Witwer KW, Aikawa E, et al. Minimal information for studies of extracellular vesicles 2018 (MISEV2018): a position statement of the International Society for Extracellular Vesicles and update of the MISEV2014 guidelines. J Extracell Vesicles. 2018;7:1535750. doi:10.1080/20013078.2018.1535750

14. Zhang R, Xie L, Wu H, et al. Alginate/laponite hydrogel microspheres co-encapsulating dental pulp stem cells and VEGF for endodontic regeneration. Acta Biomater. 2020;113:305-316. doi:10.1016/j.actbio.2020.07.012

15. Jin B, Choung PH. Recombinant human plasminogen activator inhibitor-1 accelerates odontoblastic differentiation of human stem cells from apical papilla. Tissue Eng Part A. 2016;22(9-10):721-732. doi:10.1089/ten.tea.2015.0273

16. Jiao L, Xie L, Yang B, et al. Cryopreserved dentin matrix as a scaffold material for dentin-pulp tissue regeneration. Biomaterials. 2014;35:4929-4939. doi:10.1016/j.biomaterials.2014.03.016

17. Li X, Zhang S, Zhang Z, et al. Development of immortalized Hertwig's epithelial root sheath cell lines for cementum and dentin regeneration. Stem Cell Res Ther. 2019;10:3. doi:10.1186/s13287-018-1106-8

18. Chew JRJ, Chuah SJ, Teo KYW, et al. Mesenchymal stem cell exosomes enhance periodontal ligament cell functions and promote periodontal regeneration. Acta Biomater. 2019;89:252-264. doi:10.1016/j.actbio.2019.03.021

19. Ivica A, Ghayor C, Zehnder M, Valdec S, Weber FE. Pulp-derived exosomes in a fibrin-based regenerative root filling material. J Clin Med. 2020;9 (2):491. doi:10.3390/jcm9020491

20. Phinney DG, Pittenger MF. Concise review: MSC-derived exosomes for cell-free therapy. Stem Cells. 2017;35:851-858. doi:10.1002/stem.2575

21. Chen J, Cui C, Qiao X, et al. Treated dentin matrix paste as a novel pulp capping agent for dentin regeneration. $J$ Tissue Eng Regen Med. 2017;11:3428-3436. doi:10.1002/term.2256

22. Moussa DG, Aparicio CA-O. Present and future of tissue engineering scaffolds for dentin-pulp complex regeneration. J Tissue Eng Regen Med. 2019;13:58-75. doi:10.1002/term.2769

23. Liu J, Du J, Chen X, et al. The effects of mitogen-activated protein kinase signaling pathways on lipopolysaccharide-mediated osteo/odontogenic differentiation of stem cells from the apical papilla. $J$ Endod. 2019;45(2):161-167. doi:10.1016/j.joen.2018.10.009

24. Du J, Lu Y, Song M, et al. Effects of ERK/p38 MAPKs signaling pathways on MTA-mediated osteo/odontogenic differentiation of stem cells from apical papilla: a vitro study. BMC Oral Health. 2020;20(1):50. doi:10.1186/s12903-020-1016-x

25. Liu Z, Lin Y, Fang X, Yang J, Chen Z. Epigallocatechin-3-gallate promotes osteo-/odontogenic differentiation of stem cells from the apical papilla through activating the BMP-smad signaling pathway. Molecules. 2021;26(6):1580.

26. Rombouts C, Giraud T, Jeanneau C. Pulp vascularization during tooth development, regeneration, and therapy. J Dent Res. 2017;96:137-144 doi: $10.1177 / 0022034516671688$

27. Na S, Zhang H, Huang F, et al. Regeneration of dental pulp/dentine complex with a three-dimensional and scaffold-free stem-cell sheet-derived pellet. J Tissue Eng Regen Med. 2016;10:261-270. doi:10.1002/term.1686 


\section{Publish your work in this journal}

The International Journal of Nanomedicine is an international, peer-reviewed journal focusing on the application of nanotechnology in diagnostics, therapeutics, and drug delivery systems throughout the biomedical field. This journal is indexed on PubMed Central, MedLine, CAS, SciSearch ${ }^{\circledR}$, Current Contents ${ }^{\circledR} /$ Clinical Medicine, Journal Citation Reports/Science Edition, EMBase, Scopus and the Elsevier Bibliographic databases. The manuscript management system is completely online and includes a very quick and fair peer-review system, which is all easy to use. Visit http:// www.dovepress.com/testimonials.php to read real quotes from published authors.

Submit your manuscript here: https://www.dovepress.com/international-journal-of-nanomedicine-journal 